\title{
Rotor fault detector of the converter-fed induction motor based on RBF neural network
}

\author{
C.T. KOWALSKI* and M. KAMINSKI \\ Institute of Electrical Machines, Drives and Measurements, Wroclaw University of Technology, \\ 19 Smoluchowskiego St., 50-372 Wroclaw, Poland
}

\begin{abstract}
This paper deals with the application of the Radial Basis Function (RBF) networks for the induction motor fault detection. The rotor faults are analysed and fault symptoms are described. Next the main stages of the design methodology of the RBF-based neural detectors are described. These networks are trained and tested using measurement data of the stator current (MCSA). The efficiency of developed RBF-NN detectors is evaluated. Furthermore, influence of neural networks complexity and parameters of the RBF activation function on the quality of data classification is shown. The presented neural detectors are tested with measurement data obtained in the laboratory setup containing the converter-fed induction motor (IM) and changeable rotors with a different degree of damages.
\end{abstract}

Key words: induction motor, rotor fault, diagnostic symptoms, RBF neural networks, fault detector.

\section{Introduction}

Induction motor drives are widely used nowadays in many industrial processes and thus problems of their maintenance and the fault detection become important. The failure of the motor can cause substantial financial loss and even damage of the whole drive system. Needs of the analysis of the actual technical condition of induction motors have caused recently the great development of diagnostic methods and techniques, which could be used in the fault detection of induction motors drives.

The fault statistics of high- and low-voltage induction motors has been changing within the last few years. There is a significant increase of mechanical failures in comparison with electrical and magnetic circuits failures. It can be demonstrated in the following way (as the percentage of all motor faults) $[1,2]$ :

- bearing failures $-\sim 40 \%$,

- stator failures - 36\%,

- rotor failures $-\sim 10 \%$,

- other failures $-\sim 14 \%$.

One of trends in diagnostic schemes is the model- based fault detection and isolation (FDI). Generally, symptoms of disruptions caused by induction machine damages are generated as differences between a model and an object. These proceedings provide disclosure of damages and additionally information for adaptation of parameters in controllers and observers. Such actions give a possibility of the fault tolerant drive systems design [3-5].

Important and frequently used groups of diagnostic methods of the induction motors (IM) are based on the frequency analysis of the vibration and current signals [1]. These methods should lead to early detection of faults and prevent next damages of the whole electrical drive. Usually the Fast Fourier Transformation (FFT) is used, but in the last decade the Short Time Fourier Transformation (STFT), the Wavelet Transformation (WT) and the high order transformation (HOT) are more and more often applied [6-8]. Characteristic harmonics are searched in diagnostic signals, as the faults of each part of electrical machine are showed by different frequency harmonics. The special analyzers of high harmonics or effective computers with high-resolution measurements cards are required for the precise analysis of diagnostic signals. In PWM inverter-fed electrical drives, where the frequency control of the rotor speed is realized, an additional problem connected with the changeable frequency of the supplying voltage appears, which makes the analysis of diagnostic signal spectrum more difficult $[8,9]$.

Problems eliminating the fault monitoring and detection of the inverter-fed induction motor using traditional methods enforce looking for new ways of analyzing the technical state of the electrical drive. Recently implementations of neural networks in diagnostics of IM have been very popular as well as results from very good abilities of NN's in the generalization and classification of data and also effective training of the network for solving the complex tasks [2, 10-14]. Most solutions described in technical literature are based on the multilayer perceptron MLP, with nonlinear (sigmoidal) activation functions in hidden layers. It should be noted that a training process of MLP models requires a long time and a great computing power which is disadvantageous at the stage of the NN design. Moreover, the problem associated with the selection of the NN structure appears, having significant influence on the quality of the performance realized by the designed NN. Also the training algorithm requires some parameters to be chosen in order to obtain the proper results of training. So, other than MLP networks are also tested in the IM diagnos-

*e-mail: czeslaw.t.kowalski@pwr.wroc.pl 
tics: Radial Basis Function Neural Network [15] or Learning Vector Quantization models [16].

In this paper the possibilities of application of $\mathrm{NN}$ with Radial Basis Functions in the detection of rotor faults of the converter-fed induction motor are tested. This special type of neural networks is characterized by the very fast training process and significant simplification in the design process. Moreover, two methods of the center selection of RBF NN are applied. First of them based on a direct usage of training data. In the second method the k-means clustering algorithm is implemented. This combination in the described task is innovatory.

The paper is divided into five sections. After the introduction part, the issues associated with the symptoms of the rotor fault of the IM are presented. Next the short comparison of the MLP and RBF networks is given with a focus on the design procedure of NN based fault detectors. In the subsequent parts of this paper the method of data generation with symptoms of motor defects is described and tests of the developed RBF-based fault detectors are presented. The designed detectors of rotor faults are tested basing on the measurement data obtained in the laboratory setup containing the converterfed induction motor with exchangeable rotors (with different number of broken bars). The short conclusion summarizes the presented approach and obtained results.

\section{Rotor fault symptoms in stator current spectra}

The rotor failures are among the most frequent electrical faults of IM, especially broken bars of squirrel-cage rotors. In Fig. 1 the real industrial example of such failures is shown. The main problem is connected with their destructive character and a tendency to rapid transition. Therefore, early detection of a single broken bar during motor operation would eliminate subsequent damage to adjacent bars, reducing repair costs and motor outage time.

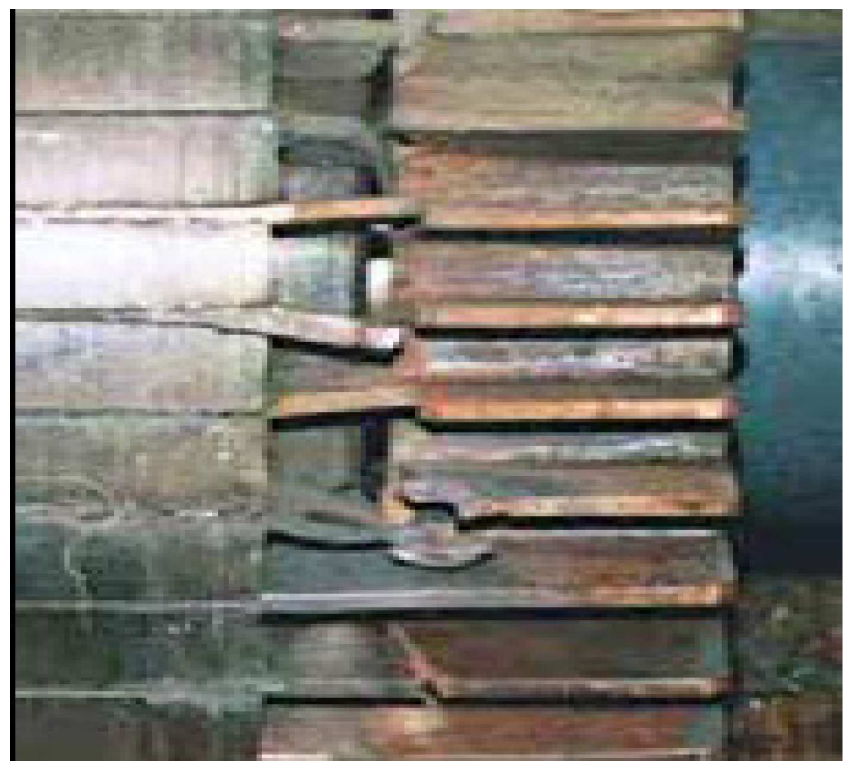

Fig. 1. Industrial example of rotor bar faults
The described faults cause the asymmetry of the magnetic field of the motor; this in turn results in various additional phenomena, such as a change of the stator current spectrum, additional internal forces and mechanical vibrations, oscillatory components of rotor speed, electromagnetic torque, output power, etc. [1]. These effects are very weak in the initial stage of the rotor fault and only sensitive measurement methods can detect the damage.

In practice, the analysis of the stator current is one of most often applied methods of non-invasive detection of the rotor asymmetry caused by broken bars [1-16]. A choice of this physical variable allows getting good diagnostic results without the necessity of the expensive measurements and thus without expansion of laboratory setup and additional cost. Characteristic components with frequency dependent on motor slip $s$ occur in the stator current spectrum in the case of the rotor bar fault. Amplitudes of harmonic components with the following, characteristic frequencies are observed under rotor fault:

- sideband harmonics around fundamental (left and right slip components), given by:

$$
\begin{aligned}
& f_{p k}^{l}=(1-2 k s) f_{s}, \\
& f_{p k}^{r}=(1+2 k s) f_{s},
\end{aligned}
$$

spatial harmonics in line current (higher order components):

$$
f_{b k}=\left[\frac{k}{p_{p}}(1-s) \pm s\right] f_{s},
$$

where $f_{s}$ - nominal frequency of stator supply voltage, $p_{p}-$ number of poles , $k=1,2,3 \ldots$

and $s-$ motor slip calculated as follows:

$$
s=\frac{n_{s}-n_{r}}{n_{s}},
$$

where $n_{s}$ - synchronous speed, $n_{r}$ - the rotor speed.

The illustration of these harmonics placement in the stator current spectrum is demonstrated in Fig. 2.

Increasing the magnitudes of stator harmonic components with characteristic frequencies is a symptom of the rotor defect, and evaluation of its level can give the information about degree of damage. However some problems in rotor fault feature extraction erase:

- changes of the load torque influence the motor slip value load changes disturb analysis of the stator current harmonic spectra,

- in case of tests realized with slight load, slip and fundamental frequencies will cover each other - a result, rotor damage diagnosis will be incorrect,

- higher harmonics can overlap with inverter supply harmonics.

As a result, the diagnosis of rotor damages will be incorrect. 
a)

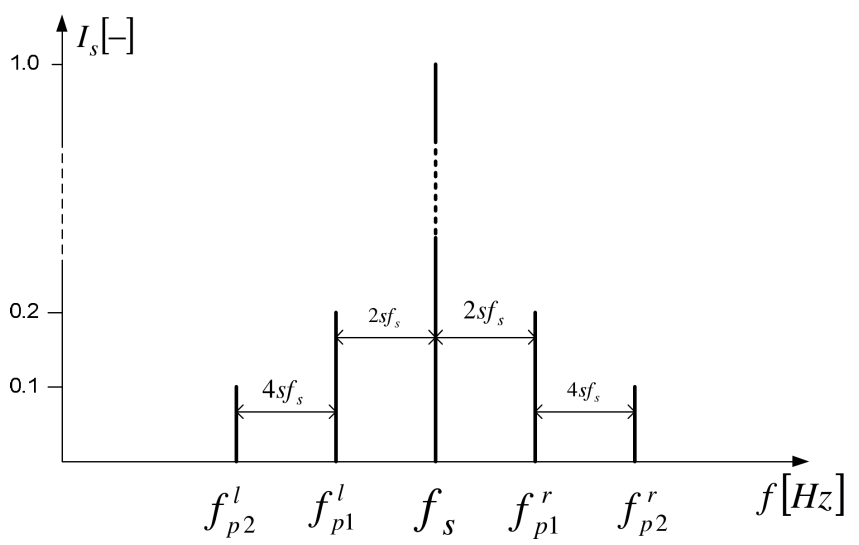

b)

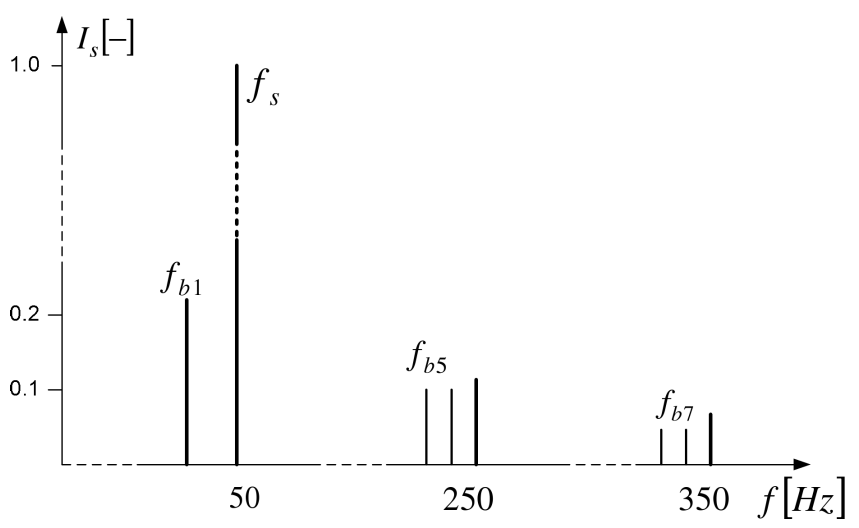

Fig. 2. Illustration of the sideband (a) and higher (b) slip harmonics placement in the stator current spectrum for broken rotor bars

The solution to this problem is using the spatial vector of stator current as an additional signal in the diagnostic procedure [7-9]. The stator current vector can be described easily by phase currents of the stator winding:

$$
i_{s}=\sqrt{\frac{2}{3}}\left(1 i_{s A}+a i_{s B}+a^{2} i_{s C}\right),
$$

where $i_{s A}, i_{s B}, i_{s C}$ - values of phase stator currents, $a=$ $e^{j \frac{2 \pi}{3}}, a^{2}=e^{j \frac{2 \pi}{3}}$.

The stator current vector of the induction motor with a healthy rotor can be transformed to a stationary coordinate system $(\alpha-\beta)$ :

$$
\begin{gathered}
i_{s \alpha}=\sqrt{\frac{2}{3}}\left(i_{s A}-\frac{1}{2}\left(i_{s B}+i_{s C}\right)\right), \\
i_{s \beta}=\frac{1}{\sqrt{2}}\left(i_{s B}-i_{s C}\right) .
\end{gathered}
$$

The magnitude of this spatial vector is calculated as:

$$
\left|i_{s}\right|=\sqrt{i_{s \alpha}^{2}+i_{s \beta}^{2}}
$$

For the symmetrical healthy rotor this modulus has a constant value. In the case of rotor fault, harmonics with characteristic frequencies appear in the $\alpha-\beta$ current components, as presented below:

$$
\begin{gathered}
i_{s \alpha}=\sqrt{\frac{3}{2}\left\{I_{m}^{s} \cos \left(\omega_{s} t-\alpha\right)+I_{p 1 l}^{s} \cos \left[(1-2 s) \omega_{s} t-\beta_{1}\right]\right.} \\
\left.\quad+I_{p 1 r}^{s} \cos \left[(1+2 s) \omega_{s} t-\beta_{2}\right]\right\} \\
i_{s \beta}=\sqrt{\frac{3}{2}\left\{I_{m}^{s} \sin \left(\omega_{s} t-\alpha\right)+I_{p 1 l}^{s} \sin \left[(1-2 s) \omega_{s} t-\beta_{1}\right]\right.} \\
\left.\quad+I_{p 1 r}^{s} \sin \left[(1+2 s) \omega_{s} t-\beta_{2}\right]\right\}
\end{gathered}
$$

where $I_{m}^{s}$ - magnitude of the stator current first harmonic, $I_{p 1 l}^{s}, I_{p 1 r}^{s}$ - magnitudes of components with frequencies:

$$
f_{p 1}^{l}=(1-2 s) f_{s} \text { and } \quad f_{p 1}^{r}=(1+2 s) f_{s} .
$$

These harmonics cause that the spectrum of the modulus of stator current vector contains other specific components:

$$
f_{\nu k}=2 k s f_{s}, \quad k=1,2, \ldots
$$

with frequencies $2 s f_{s}$ and $4 s f_{s}$ and so on.

These harmonics are very good symptoms of the rotor defects, as they are much further away from the basic current harmonic than the slip harmonics (1), and assumes much higher values. This is illustrated in Fig. 3.

a)

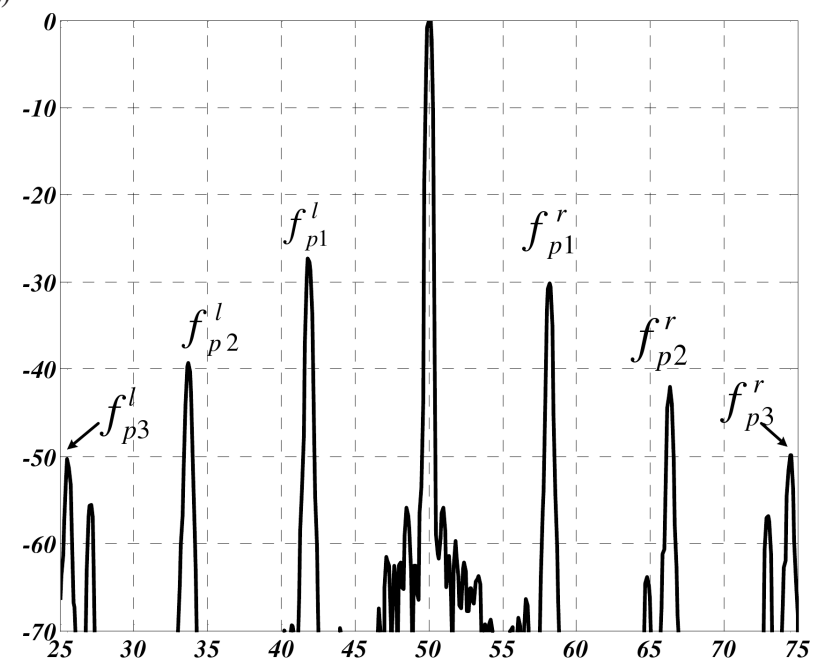

b)

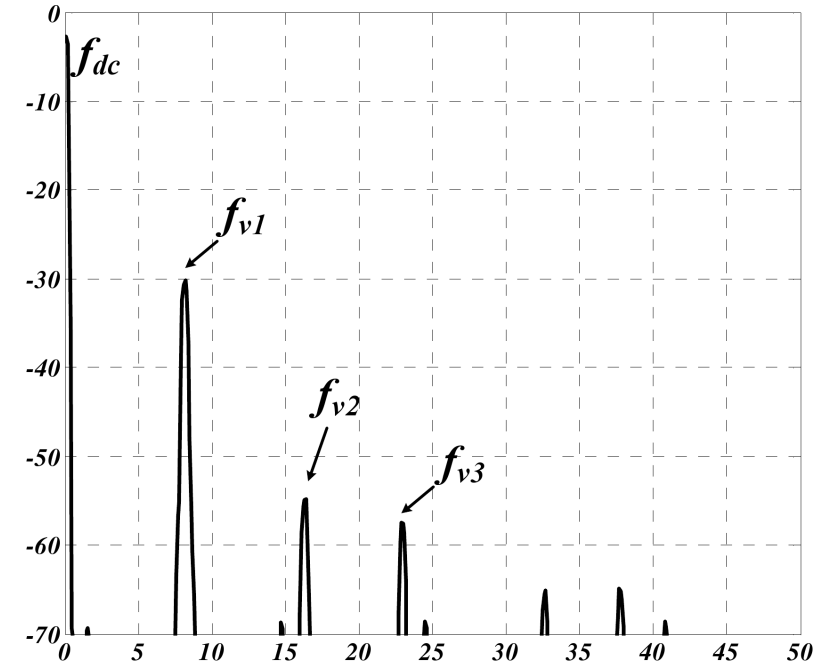

Fig. 3. Differences in faults symptoms for stator current (a) and spatial current vector (b) spectra 
The above relationships describing the characteristic frequencies of the stator current in the case of rotor damages enable the calculation of fault symptoms for design of input vectors for detectors based on neural networks.

\section{Neural networks with Radial Basis Functions}

The RBF (Radial Basis F unction) networks are special types of neural networks with characteristic processing rules and different activation functions in the hidden layer than MLP (Multi Layer Perceptron) networks [17].

MLP neural networks with the nonlinear sigmoidal activation functions perform a global approximation task. Projection of several elements of input vector in this case is dependent on many neurons at the same time. On the contrary, for RBF network, projection of one cluster from the data set is realised by one hidden neuron, which takes into account the hyper spherical-shaped clusters of data concentrated around the central point. Such an approach to the problem signifies the local approximation.

The RBF network is a three-layer feed-forward neural network, as shown in Fig. 4. It consists of one input layer, one hidden and one output layer.

a)

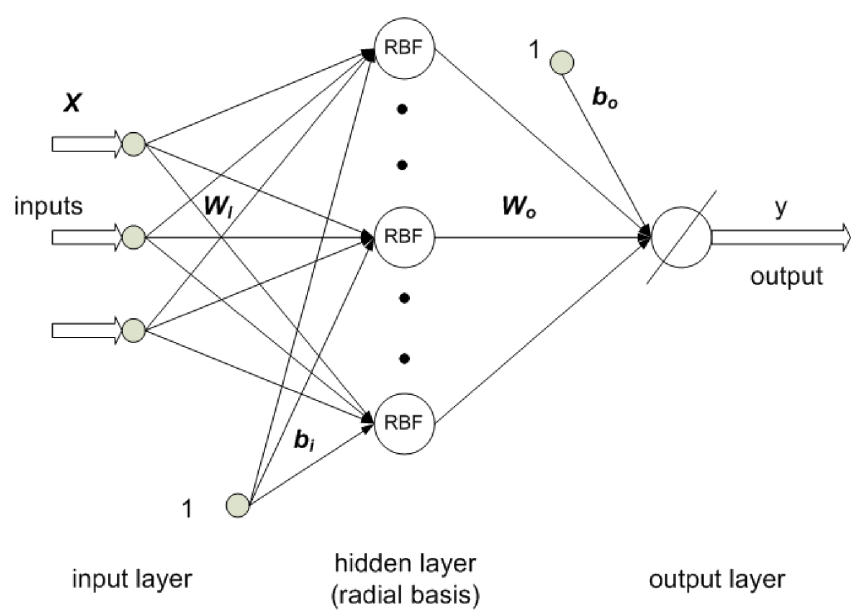

b)

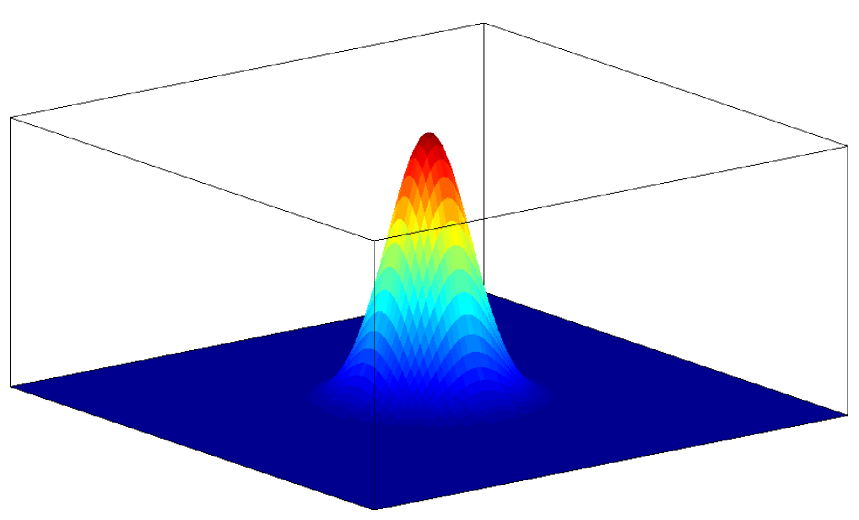

Fig. 4. Structure of the RBF neural network (a), Gauss activation function for 2-dimensional input data (b)
Each input neuron corresponds to an element of an input vector and is fully connected to the $n$ hidden layer neurons, excepting the bias connection. Again, each of the hidden layer neurons and the bias neuron are also fully connected to the output neurons. 'Fully connected' means in the described case connection of each node in a layer to all neurons in the next layer (without pruned weights). Hidden layer includes neurons with radial basis activation functions. For the hidden layer the Gauss activation functions are mostly applied [17, 18]. Output layer consists of neurons with linear activation function and has the same number of neurons as a size of output signal of the network. Specific structure and technique of signal processing in RBF networks make possible the reconstruction of any nonlinear function by one hidden layer. However, it is necessary to implement much higher number of neurons than in MLP nets. However the design process for RBF networks is much simpler than for MLP.

The mapping from input to output is nonlinear, but from hidden layer to output layer is linear. Learning rate is much shorter and the problem of local minimum is avoided.

The learning procedure of RBF network consists of three stages that include:

- choice of the centres of the hidden radial basis neurons,

- choice of parameter $\sigma$ - width of the radial function for each hidden neuron,

- determination of the weight factors between hidden (radial) and output layer.

In the first stage usually the Euclidean distance between input vector values and centres of RBF functions is calculated:

$$
\begin{gathered}
v_{j}(\mathbf{X})=\left\|\mathbf{X}-\mathbf{C}_{j}\right\|=\sqrt{\sum_{k=1}^{N}\left(x_{k}-c_{k}\right)^{2}}, \\
k=1,2,3 \ldots, N,
\end{gathered}
$$

where $\mathbf{X}=\left[x_{1}, x_{2}, x_{3}, \ldots, x_{N}\right]^{T}$ is the input vector, $\mathbf{C}_{j}-$ vector related to the centre of each neuron.

Next, parameters $v_{j}(\mathbf{X})$ are scaled by multiplication of their values by bias values. Vector of biases has constant value for each neuron:

$$
b_{i}=b=\left(\frac{\sqrt{-\log (0,5)}}{\sigma}\right),
$$

where $\sigma-$ is a spread factor of the RBF neuron.

In this way the input signals for RBF neurons are obtained:

$$
h_{j 1}=h\left(\zeta_{j k}\right)=\exp \left(-\left(v_{j k} b_{j 1}\right)^{2}\right) .
$$

Output values of the network are obtained as sum of product of the RBF neurons' outputs and weight factors:

$$
y_{k}=\sum_{j=1}^{N} w_{j k} h_{j}+b_{k},
$$

where $y_{k}-k$-th output of the network, $w_{j k}$ - weight factor between the $j$-th output of the hidden (RBF) layer and $k$-th neuron of NN output, $b_{k}$ - bias of $k$-th output neuron. 
Determination of the weight factors in the output layer can be performed using the following formula [18]:

$$
\mathbf{W}=\left(\mathbf{H}^{T} \mathbf{H}\right)^{-1} \mathbf{H}^{T} \mathbf{R},
$$

where

$$
H_{i j}=h_{j 1}\left(\zeta_{j k}\right) .
$$

In the presented work the placement of radial neuron centers was performed using two methods:

- the direct assignment of all elements of the training data set as cluster centers,

- K-means method [19].

In practice, in this stage of design process, complexity of neural network is determined. Structure of neural model has influence on two main aspects: generalization features and hardware implementation. Generalization defines ability of neural network to realization of some task based on data not used in training. It is important in real applications, where training database is often limited. If number of neurons in neural network is too small, it can be not enough for realization of task. Also when structure is too extended generalization is not optimal. Selection of neural network size has influence on calculation time and hardware resources utilization (FPGA) during real application. It should be noted that in most application RBF network has higher number of hidden neurons (comparing to MLP).

Application of the first method mentioned above significantly simplifies the design process of the RBF network. But in this case the data set is divided into the relatively large number of clusters, which contain all data elements. It results in a very complex NN structure, with large number of neurons in the hidden layer. It implies complex calculations connected with very large numbers of elements of the weight matrix and does not guarantee the generalization features of the network. Moreover, the practical implementation of such extensive NN architecture is very difficult and usually not possible [20].

The K-means method represents the algorithm of data clustering. Calculations are conducted using iterative procedure. After the selection of initial cluster centers they are updated as the average of the members of the corresponding cluster using point symmetry distance measure, usually based on the Euclidean norm. Updating of cluster centers terminates when there are no significant changes of cluster centers in two consecutive iterations, giving $K$ final cluster centers [19]. Such method guarantees a convergence of the calculations and minimization of the number of RBF neurons in the hidden layer.

\section{Generation of rotor fault symptoms for the induction motor drive}

In the diagnostic process of the induction motor the following stages can be distinguished:

- measurements of physical quantities of a healthy and faulted motor, using special sensors,

- preprocessing of measurement data for fault severity,

- classification of the obtained data and fault detection with RBF neural networks.

The converter-fed induction motor of $3 \mathrm{~kW}$ nominal power and nominal speed $n=1400 \mathrm{rpm}$ was tested. The DC motor with suitable supply system was used as a loading machine. During the measurements five values of the load torque from the range $0-125 \%$ of its nominal value were applied to the driving motor. Measurements were performed using LEM current sensors and NI PXI-4461 data acquisition card, as in Fig. 5.

For data processing of the current signals the Fast Fourier Transform (FFT) was applied. In the analyzed case the measurements were made with the frequency resolution: $d f=$ $0.048\left(2^{19}\right.$ samples in the time sequence $\left.20.97 \mathrm{~s}\right)$ in the range of $25 \mathrm{kHz}$.

Based on the conducted measurements and data processing the following set of diagnostic features for the NN input vector was proposed:

$$
\mathbf{X}=\left(I_{\max }, s_{l 1}, s_{r 1}, s_{l r 1}, p_{1}, p_{2}\right),
$$

where $I_{\max }$ - magnitude of zero component of the modulus of the stator current spatial vector, $s_{l 1}, s_{r 1}-$ left and right magnitudes of the slip components $\left(f_{p k}^{l, r}, k=1\right)$ of the stator current, related to the fundamental current harmonic, $s_{l r 1}-$ sum of components $s_{l 1}$ and $s_{r 1}, p_{1}, p_{2}$ - magnitudes of fault components $\left(f_{\nu k} ; k=1,2\right)$ extracted from the modulus of the stator current spatial vector, related to the magnitude of this vector $I_{\max }$.

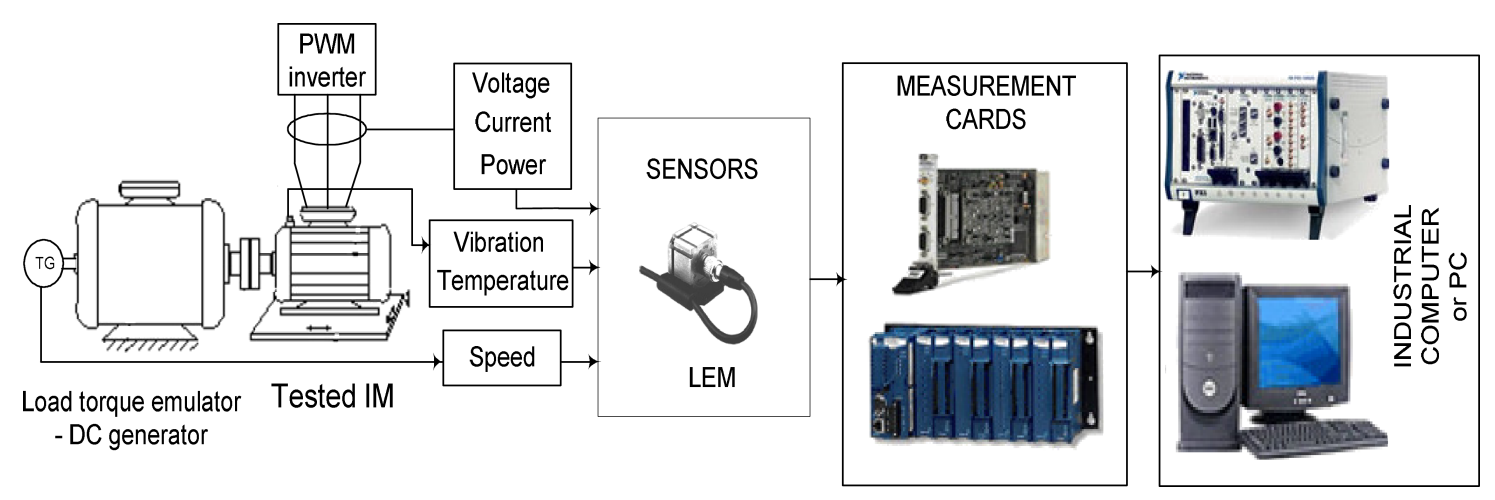

Fig. 5. Schematic diagram of the laboratory test rig 
Data sets for training and testing of NN detectors were obtained from experimental measurements of the IM with exchangeable rotors, with different degree of damage. An extensive database contained 900 records was created. The data set was divided into three parts; two of them were used for training (and contained 600 input records), the third - containing 300 records - was used for testing of the designed detectors.

\section{Test results}

In experimental tests both design methods (direct and Kmeans) were used. To compare the achieved results, a percentage effectiveness of the detected number of broken rotor bars in the examined number of measured samples was assumed. The quality assessment was taken from means value of the calculated effectiveness of detection. Testing results for both design methods are compared in Fig. 6.

a)

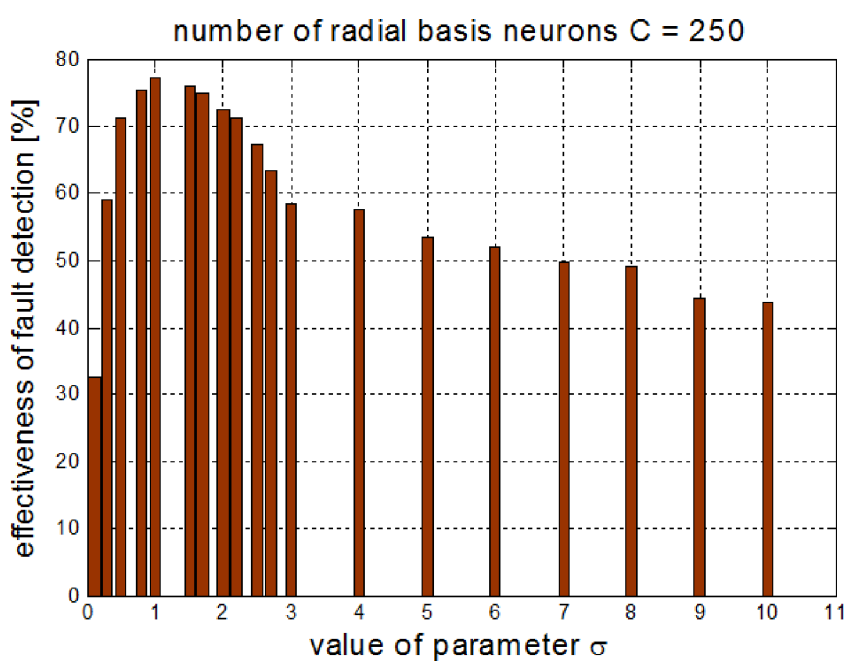

b)

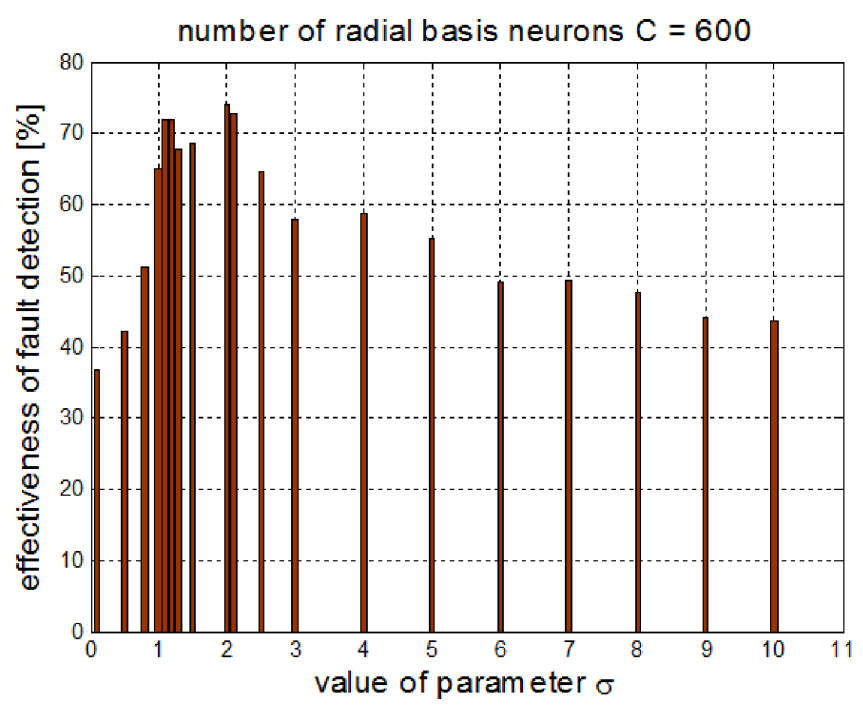

Fig. 6. Dependence of the fault detection effectiveness on the parameter $\sigma$ of the RBF-NN
Very good accuracy of the fault detection was achieved for measurements conducted for loaded motor supplied from the static converter. It is seen that parameter $\sigma$ of the radial basis neurons has significant influence on this accuracy (Fig. 6). The value of parameter $\sigma$ determines the bias value in the $\mathrm{RBF}$ layer and thus influences the input signals of the following output neurons also. The best effectiveness of tested $\mathrm{RBF}$ detectors is obtained for the bigger range of variations of the radial activation function in the hidden layer neurons. It can be seen also that parameter $\sigma$ influences the detection effectiveness in the similar way while using K-means and the direct methods, but in case of the first one, the same detection quality can be obtained with much smaller number of $\mathrm{RBF}$ hidden neurons which is very important under practical implementation of such detectors.

In the case of detector designed with K-means method, the influence of the number of hidden neurons to the rotor fault detection accuracy was tested and results are shown in Fig. 7. All tests were conducted for constant value of coefficient $\sigma=1.2$. It is seen that hidden neurons' number has significant influence on detection quality. The best detection results (close to $78 \%$ proper diagnoses) for the tested motor were obtained with the network containing 250 hidden RBF neurons. For the smaller number of hidden neurons the network is not able to perform proper mapping of the input data. On the other hand, for the bigger number of hidden neurons the network looses its generalization abilities (Fig. 7).

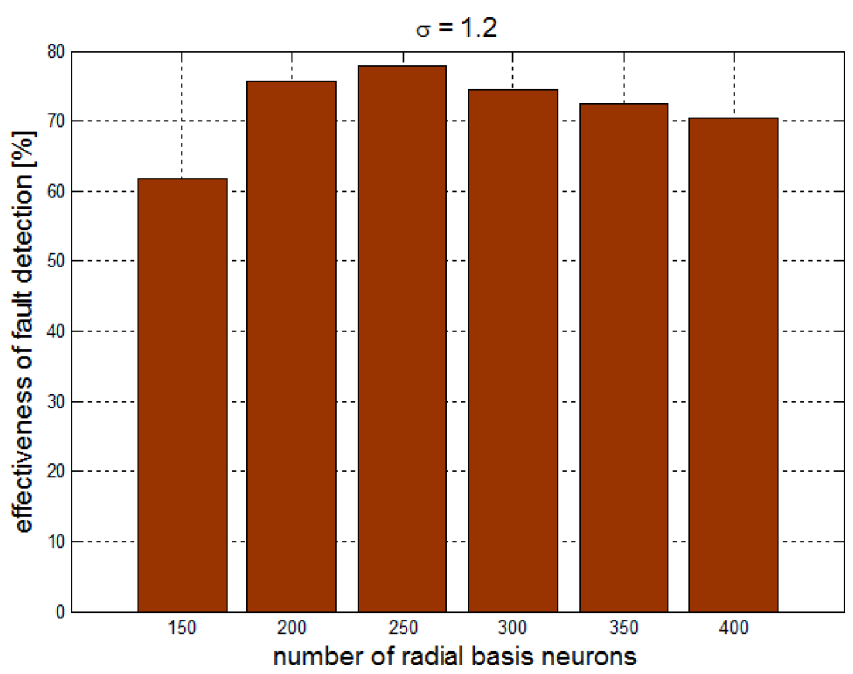

Fig. 7. Influence of the hidden neurons number of the RBF network to the effectiveness of rotor fault detection using K-means method

Next, obtained results of the detection of faulted rotor bars number are demonstrated in Fig. 8 for both design methods of RBF networks. Figures $8 \mathrm{a}$ and $8 \mathrm{c}$ show values of broken rotor bars calculated by $\mathrm{NN}$, and Figs. $8 \mathrm{~b}$ and $8 \mathrm{~d}$ show the rounded values, respectively. It is seen from the presented results, that the application of K-means method for determination of centers of the RBF neurons ensures better accuracy of the fault detection (78\%) than the results obtained with the direct method $(74 \%)$. 
a)

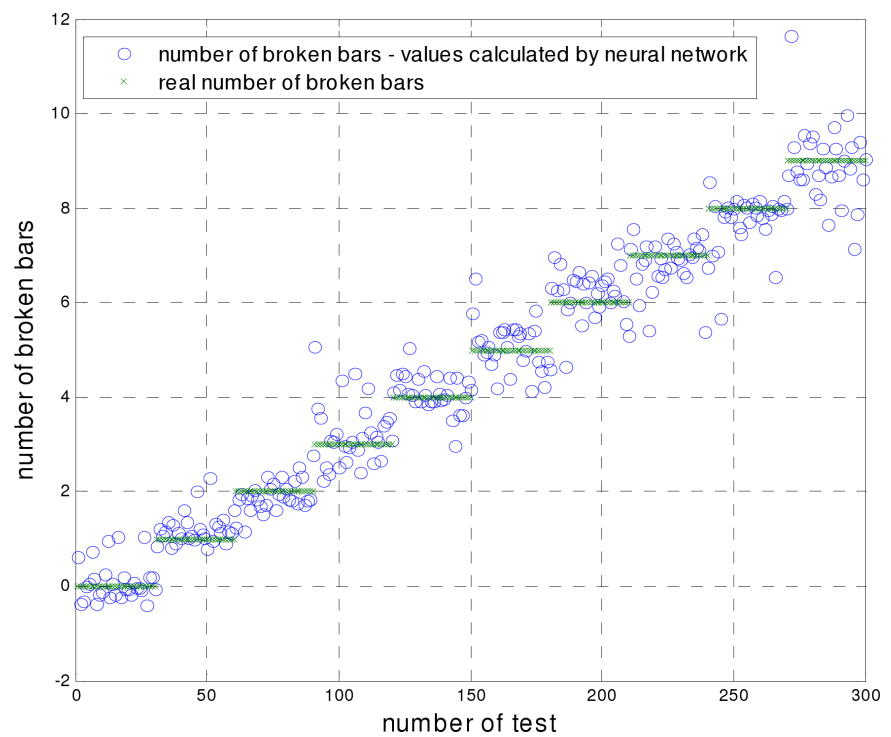

c)

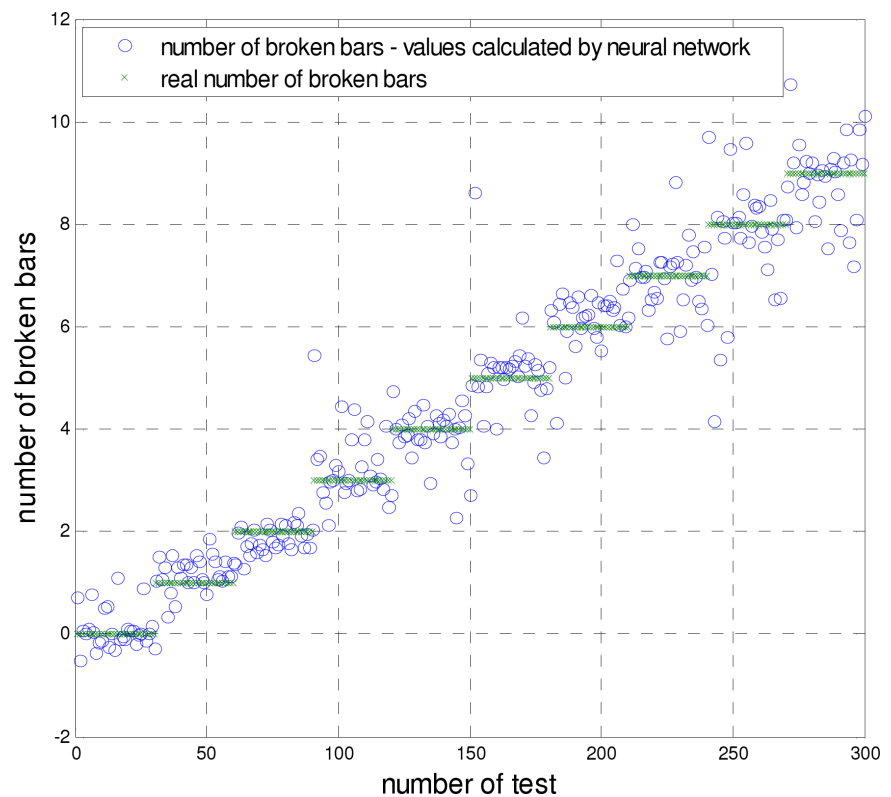

b)

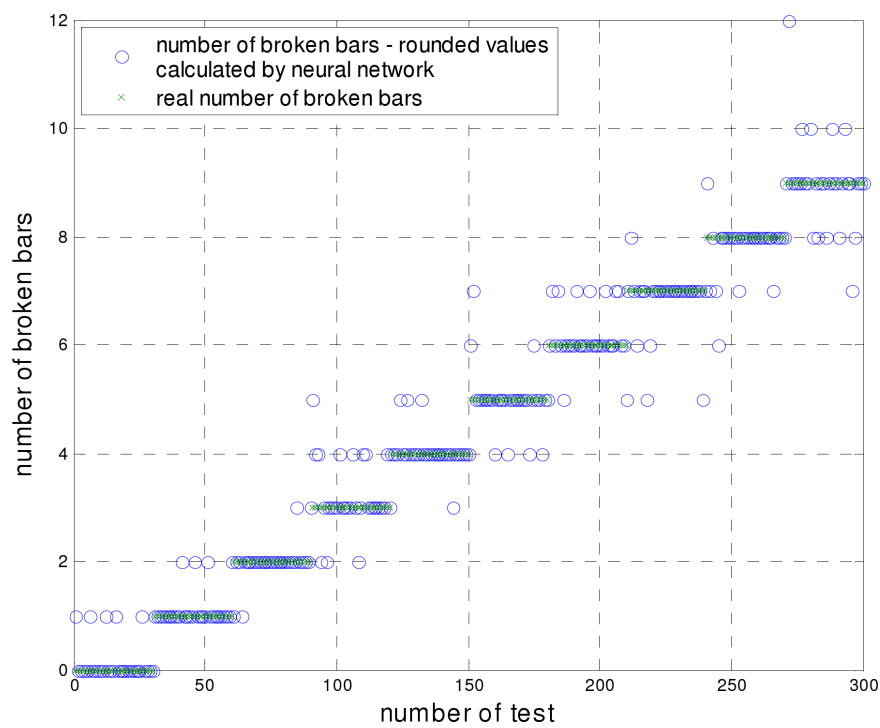

d)

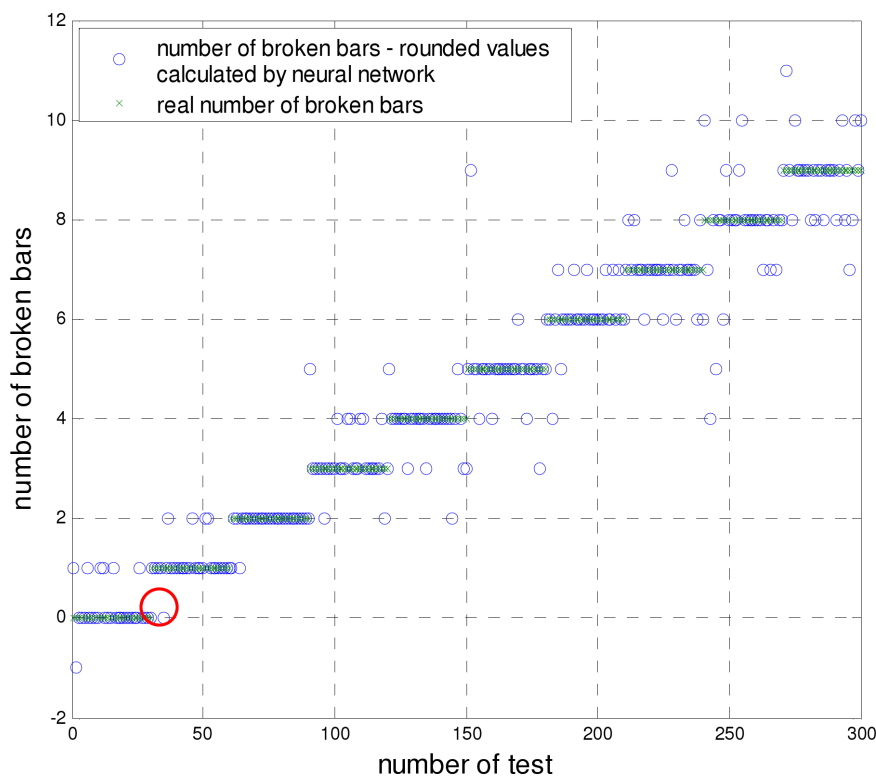

Fig. 8. Influence of the design method of the RBF neurons to the detection quality of the number of broken rotor bars of the induction motor: (a), (b) - K-means-methods, (c), (d) - direct method (a), (c) - exact calculation values, (b), (d) - rounded values

When analyzing the obtained results from the point of view of the IM diagnostics, especially in the case of converter supply, it can be stated that the obtained accuracy is very good. Moreover, in the case of the best developed detectors, we have no wrong diagnoses in the case when rotor has only one broken bar (see Fig. 8a,b). On the contrary, for the direct design method of the RBF neurons sometimes wrong responses of the NN are obtained, eg. the rotor is healthy when in fact it contains one broken bar (see Fig. 8c,d). It should be mentioned that all demonstrated results were obtained for the expanded data set of measurements, acquired for different motor loads and different supplying frequencies.

\section{Conclusions}

Basing on the presented results, following conclusions can be formulated:

- when analyzing the obtained results from the point of view of the IM diagnostics, especially in the case of converter supply, it can be stated that the obtained accuracy is very good;

- moreover, in the case of the best developed detectors, using $\mathrm{K}$-means method, we have no wrong diagnoses in the case when a rotor has only one broken bar; 
- on the contrary, for the direct design method of the RBF neurons, sometimes the NN responds that the rotor is healthy when in fact it contains one broken bar;

- very good results for the IM rotor fault detection using RBF neural networks are obtained in the presence of the load changes and converter supply harmonics, for the relatively short input vector of $\mathrm{NN}$;

- a very simple process of the RBF networks design is their most valuable advantage and RBF-based detectors can be an alternative to the well-known MLP-based fault detectors.

\section{REFERENCES}

[1] W.T. Thomson, "A review of on-line condition monitoring techniques for three-phase squirrel-cage induction motors past, present and future", Proc. IEEE Int. Symp. SDEMPED 1, 3-18 (1999).

[2] C.T. Kowalski and T. Orlowska-Kowalska, "Application of neural networks for the induction motor faults detection", Mathematics and Computers in Simulation - Trans. IMACS 63 (3-5), 435-448 (2003).

[3] C.J. Lopez-Toribio, R.J. Patton, and S. Daley, "A mutiplemodel approach to fault-tolerant control using Takagi-Sugeno fuzzy modelling: real application to an induction motor drive system", Eur. Control Conf., ECC 99 1, CD-ROM (1999).

[4] K.S. Gaeid and H.W. Ping, "Induction motor fault detection and isolation through unknown input observer", Scientific Research and Essays 5 (20), 3152-3159 (2010).

[5] C.J. Lopez-Toribio, R.J. Patton, and S. Daley, "Takagi-Sugeno fuzzy fault-tolerant control of an induction motor", Neural Computing \& Applications 9 (1), 19-28 (2000).

[6] M. Benbouzid, "A Review of induction motors signature analysis as a medium for faults detection", IEEE Trans. on Ind. Electronics 47 (5), 984-993 (2000).

[7] M. Cruz and A.J. Cardoso, "Rotor cage fault diagnosis in threephase induction motors by extended park's vector approach", Electric Machines and Power Systems 28 (4), 289-99 (2000).

[8] C.T. Kowalski and M. Pawlak, "Application of the current space vector method for detection of induction motor faults", Przegląd Elektrotechniczny 79 (7/8), 771-777 (2004).
[9] C. Bruzzese, "Analysis and application of particular current signatures for cage monitoring in non-sinusoidally fed motors with high rejection to drive load, inertia, and frequency variations", IEEE Trans. on Ind. Electronics 55 (12), 4137-4155 (2008).

[10] B. Akin, U. Orguner, H.A. Toliyat, and M. Rayner, "Low Order PWM inverter harmonics contributions to the inverter-fed induction machine fault diagnosis", IEEE Trans. on Industrial Electronics 55 (2), 610-619 (2008).

[11] M.A. Awadallah and M.M. Morcos, "Application of AI tools in fault diagnosis of electrical machines and drives - an overview", IEEE Trans. on Energy Conversion 18 (2), 245251 (2003).

[12] F. Filippetti, G. Frenceschini, C. Tassoni, and P. Vas, "Recent developments of induction motor drives fault diagnosis using AI techniques", IEEE Trans. on Industrial Electronics 47 (5), 994-1004 (2000).

[13] C.T. Kowalski and M. Pawlak, "Application of AI methods for rotor faults detection of the induction motor", Acta Electrotechnica et Informatica 1, 39-41 (2004).

[14] H. Su and K.T. Chong, "Induction machine condition monitoring using neural network modeling", IEEE Trans. Industrial Electronics 54 (1), 241-249 (2007).

[15] A. Sobolewski, "Application of neural classifiers", PHD Dissertation, Bialystok University of Technology, Białystok, 2008, (in Polish).

[16] A. Sobolewski, "Neural classifiers of fault symptoms in induction machinery rotor fault diagnosis", Diagnostics 35, 27-30 (2005).

[17] M.C. Bishop, Neural Networks for Pattern Recognition, Oxford University Press, Oxford, 1996.

[18] M.J.L. Orr, "Recent advances in radial basis function networks", in Technical Report, Institute for Adaptive and Neural Computation, Univ. of Edinburgh, Edinborough, 1999.

[19] T. Kanungo, D.M. Mount, N. Netanyahu, C. Piatko, R. Silverman, and A.Y. Wu, "An efficient k-means clustering algorithm: analysis and implementation", IEEE Trans. on Pattern Analysis and Machine Intelligence 24 (7), 881-892 (2002) .

[20] T. Orlowska-Kowalska and M. Kaminski, "FPGA implementation of the multilayer neural network for speed estimation of the two-mass drive system", IEEE Trans. on Industrial Informatics 7 (3), 436-445 (2011). 